\title{
The surface electric field of bare strange stars
}

\author{
J. $\mathrm{Hu}^{1}$ and R. X. $\mathrm{Xu}^{2,1}$ \\ 1 Center for Astrophysics and Physics Department, Tsinghua University, Beijing 100084, PR China \\ 2 School of Physics, Peking University, Beijing 100871, PR China \\ Received 7 January 2002 / Accepted 4 March 2002

\begin{abstract}
The surface electric characteristics of bare strange stars are investigated with the inclusion of boundary effects. The thickness of the electron layer where pairs can be spontaneously created is calculated as a function of the bag parameters. We find that previous estimates are representative for bag parameters within a rather wide range, and therefore our results favor the thermal radiation mechanism of bare strange stars advanced by V. V. Usov.
\end{abstract}

Key words. radiation mechanisms: thermal - elementary particles - stars: neutron

\section{Introduction}

It is of great importance to identify strange stars; a new window to distinguish neutron stars and bare strange stars (BSSs) has been proposed recently based on their sharp differences in surface conditions (Xu et al. 2001; Usov 2001a). It is therefore essential to study the surface properties of BSSs in detail, e.g., the degree of the thermal luminosity of a hot BSS.

The surface electric field should be very strong $\left(\sim 10^{17} \mathrm{~V} / \mathrm{cm}\right)$ near the bare quark surface of a strange star because of the mass difference of the strange quark and the up (or down) quark (Alcock et al. 1986), which could play an important role in producing the thermal emission of BSSs by the Usov mechanism (Usov 1998; Usov 2001b), because strange quark matter is a poor radiator of thermal photons at frequencies lower than its plasma frequency $(\sim 20 \mathrm{MeV})$ (Alcock et al. 1986). The basic idea of the Usov mechanism is that $\mathrm{e}^{ \pm}$pairs are created rapidly in a few empty quantum states with energy $\epsilon<\epsilon_{\mathrm{F}}-2 m c^{2}\left(\epsilon_{\mathrm{F}}\right.$ is the Fermi energy, $m$ the electron mass) due to the very strong electric field in an electron layer (with a height of $\sim 500 \mathrm{fm}$ above quark surface); the pairs subsequently annihilate into photons which are then thermalized in the electron layer ${ }^{1}$ around a BSS. This radiative mechanism recently has been applied tentatively to soft $\gamma$ ray repeaters (Usov 2001c; Usov 2001d). In addition, the strong electric field plays an essential role in forming a possible crust around a strange star, which has been investigated extensively by many authors (e.g., Martemyanov 1992; Kettner et al. 1995; Huang \& Lu 1997; Phukon 2000;

Send offprint requests to: R. X. Xu,

e-mail: rxxu@bac.pku.edu.cn

1 This layer may be optically thick for BSSs with temperature $T \gtrsim 10^{9} \mathrm{~K}$. see Zdunik et al. 2001 for the recent developments with the inclusion of rotating and general-relativistic effects). Also it should be noted that this electric field has some important implications on pulsar radio emission mechanisms (Xu \& Qiao 1998; Xu et al. 1999; Xu et al. 2001).

In fact the Usov mechanism of pair production depends on many parameters; it is therefore imperative to study the dependence of the process on these parameters. With some typical parameters chosen by Usov in his calculations, the resultant thickness of the electron layer with the electric field $E \gtrsim 1.3 \times 10^{16} \mathrm{~V} / \mathrm{cm}$ (the critical field necessary for pair production), $\Delta r_{\mathrm{E}}$, is $\sim 500 \mathrm{fm}$. However the proper determination of the thickness $\Delta r_{\mathrm{E}}$ should be done with the dynamical theory of the strange quark matter. Because of the intractable nature of quantum chromodynamics, some phenomenological models, i.e., the MIT bag model (e.g., Jensen \& Madsen 1996), the quark mass-density-dependent model (e.g., Lugones \& Benvenuto 1995), and the quark potential model (e.g., Dey et al. 1998), have been applied to the descriptions of the strange quark matter. In the bag model, $\Delta r_{\mathrm{E}}$ is a function of $\alpha_{\mathrm{c}}$ (the coupling constant for strong interactions), $m_{\mathrm{s}}$ (the strange quark mass), and $B$ (the bag constant). Also it should be noticed that the quark number densities, $n_{\mathrm{i}}(\mathrm{i}=\mathrm{u}, \mathrm{d}$, s for up, down and strange quarks, respectively), are assumed to be uniform below the quark surface, and therefore the quark charge density $\left(2 n_{\mathrm{u}}-n_{\mathrm{d}}-n_{\mathrm{s}}\right) / 3=V_{\mathrm{q}}^{3} /\left(3 \pi^{2}\right)$ is constant near the surface. The potential $V_{\mathrm{q}}$ is usually chosen as $20 \mathrm{MeV}$ for typical cases. However, the quark number densities should not be uniform near the surface if boundary effects are included, since charge neutrality is broken there.

In this paper we improve the calculation of the electric field in the vicinity of a BSS surface, using the popular bag model and the Thomas-Fermi model. We investigate the 
electric characteristics of BSSs for different values of $\alpha_{\mathrm{c}}$. Initially we use typical parameters for $m_{\mathrm{s}}=200 \mathrm{MeV}$ and $B^{1 / 4} \sim 145 \mathrm{MeV}$. Then the thickness, $\Delta r_{\mathrm{E}}$, of the electron layer where pairs can be created is computed as functions of $B, m_{\mathrm{s}}$, and $\alpha_{\mathrm{c}}$. It is found that Usov's estimates are representative for bag-model parameters within a rather wide range. Boundary effect are also considered in this calculation.

\section{Calculation of the electric field near the quark surface}

The interesting electric properties of strange stars were first noted by Alcock et al. (1986), who presented numerical calculations of the electric potentials of strange stars with or without crusts. Analytical solutions of the electron number density and the electric field were also formulated, which could be helpful in dealing with some physical processes near the quark surfaces of strange stars (Xu \& Qiao 1999). In this section, the bag model is applied to calculate the quark charge density; the Thomas-Fermi Model is employed to find $\Delta r_{\mathrm{E}}$ dependence on $\alpha_{\mathrm{c}}, m_{\mathrm{s}}$, and $B$, with the inclusion of boundary effects.

\subsection{Equations}

In our calculation, the quarks and electrons near the surface of the strange star keep chemical equilibrium locally; the relation between charge density and electric potential is described by the classical Poisson equation. The thermodynamic potentials $\Omega_{\mathrm{i}}$ as functions of chemical potentials $\mu_{\mathrm{i}}(\mathrm{i}=\mathrm{u}, \mathrm{d}, \mathrm{s}, \mathrm{e}), m_{\mathrm{s}}$ and $\alpha_{\mathrm{c}}$ can be found in the literature (Alcock et al. 1986). In the chemical equilibrium of weak interaction,

$\mu_{\mathrm{d}}=\mu_{\mathrm{s}}=\mu$,

$\mu_{\mathrm{e}}+\mu_{\mathrm{u}}=\mu$,

$\frac{\mathrm{d}^{2} V}{\mathrm{~d} z^{2}}=n_{\mathrm{e}}+\frac{1}{3} n_{\mathrm{d}}+\frac{1}{3} n_{\mathrm{s}}-\frac{2}{3} n_{\mathrm{u}}$,

$n_{i}=-\frac{\partial \Omega_{i}}{\partial \mu_{i}}$

where $z$ is a measured height above the quark surface.

The quark number densities drop to zero on the surface, but they are not uniform below the surface if the boundary effects are considered. The chemical potential $\mu$ can be determined by the condition that the pressure on the quark surface is zero, $\left.P\right|_{z=0}=0$. The pressure is $P=-\sum_{i} \Omega_{i}-B$ (Alcock et al. 1986).

The kinetic energy of electrons is equal to the electric potential, $p_{\mathrm{e}}=V$, in the Thomas-Fermi model. The electron number density reads (Alcock et al. 1986)

$n_{\mathrm{e}}=\frac{p_{\mathrm{e}}^{3}}{3 \pi^{2}}=\frac{V^{3}}{3 \pi^{2}}$

Note that the thermodynamical potential $\Omega_{i}$ is defined when the electric potential $V$ is assumed as zero. We thus
Table 1. The particle number densities far below the quark surface, and the potentials of $V_{0}=V(z=-\infty)$ and $V_{\mathrm{c}}=$ $V(z=0)$, for different values of $\alpha_{\mathrm{c}}$.

\begin{tabular}{c|cccccc}
\hline \hline$\alpha_{\mathrm{c}}$ & 0 & 0.1 & 0.3 & 0.5 & 0.7 & 0.9 \\
\hline$B^{1 / 4} / \mathrm{MeV}$ & 146 & 144 & 139 & 133 & 127 & 120 \\
\hline$n_{\mathrm{u}} / 10^{44} \mathrm{~m}^{-3}$ & 2.86 & 2.71 & 2.41 & 2.10 & 1.80 & 1.48 \\
\hline$n_{\mathrm{d}} / 10^{44} \mathrm{~m}^{-3}$ & 3.95 & 3.68 & 3.14 & 2.60 & 2.07 & 1.54 \\
\hline$n_{\mathrm{s}} / 10^{44} \mathrm{~m}^{-3}$ & 1.77 & 1.74 & 1.68 & 1.61 & 1.52 & 1.42 \\
\hline$n_{\mathrm{e}} / 10^{40} \mathrm{~m}^{-3}$ & 13.9 & 11.9 & 7.77 & 4.03 & 1.21 & 0.30 \\
\hline$V_{0} / \mathrm{MeV}$ & 31.65 & 29.99 & 26.05 & 20.93 & 14.01 & 4.08 \\
\hline$V_{\mathrm{c}} / \mathrm{MeV}$ & 30.69 & 29.11 & 25.34 & 20.44 & 13.77 & 4.06 \\
\hline
\end{tabular}

replace $\mu_{i}$ with $\mu_{i}-q V$ in Eq. (4), where $q$ is the particle charges. Finally we come to,

$$
\frac{\mathrm{d}^{2} V}{\mathrm{~d} z^{2}}= \begin{cases}\frac{V^{3}}{3 \pi^{2}}+\frac{1}{3} n_{\mathrm{s}}(V)+\frac{1}{\pi^{2}}\left[\frac{1}{3}\left(\mu+\frac{1}{3} V\right)^{3}\right. & \\ \left.-\frac{2}{3}\left(\mu-\frac{2}{3} V\right)^{3}\right]\left(1-\frac{2 \alpha_{\mathrm{c}}}{\pi}\right), & z<0 \\ \frac{V^{3}}{3 \pi^{2}}, & z \geq 0\end{cases}
$$

where the complex term $n_{\mathrm{s}}(V)$ can be derived from chemical potentials the $\Omega_{\mathrm{i}}$.

The boundary conditions for Eq. (6) are

$z \rightarrow-\infty: \quad V \rightarrow V_{0}, \quad \mathrm{~d} V / \mathrm{d} z \rightarrow 0$

$z \rightarrow+\infty: \quad V \rightarrow 0, \quad \mathrm{~d} V / \mathrm{d} z \rightarrow 0$.

where $V_{0}$ is the electric potential in the deep core of the strange star, which is determined from Eq. (6) by letting the left hand side equal zero.

\subsection{Results}

By integrating Eq. (6) in $\left(-\infty, V_{\mathrm{c}}\right]$ and $\left[V_{\mathrm{c}},+\infty\right)$ respectively with the boundary conditions, we get the expression of the electric field $E=-\mathrm{d} V / \mathrm{d} z$, where $V_{\mathrm{c}}=\left.V\right|_{z=0}$. Obviously $V_{\mathrm{c}}$ is a function of $\alpha_{\mathrm{c}}$, which can be obtained from the continuity of $E$ at $z=0$. Substituting $V_{\mathrm{c}}$ into the equations of $E$ and then integrating them, we get the expression of $V$. For $z \geq 0$, we find

$$
\begin{aligned}
& V(z)=\frac{V_{\mathrm{c}}}{1+\frac{V_{\mathrm{c}} z}{\sqrt{6} \pi}}, \\
& E(z)=-\frac{\mathrm{d} V}{\mathrm{~d} z}=\frac{1}{\sqrt{6} \pi} \frac{V_{\mathrm{c}}^{2}}{\left(1+\frac{V_{\mathrm{c}} z}{\sqrt{6} \pi}\right)^{2}}, \\
& n_{\mathrm{e}}(z)=\frac{V^{3}}{3 \pi^{2}}=\frac{1}{3 \pi^{2}} \frac{V_{\mathrm{c}}^{3}}{\left(1+\frac{V_{\mathrm{c}} z}{\sqrt{6} \pi}\right)^{3}} .
\end{aligned}
$$

Several numerical results are obtained, which are shown in Table 1 and Figs. 1-4. First, we study the electric 


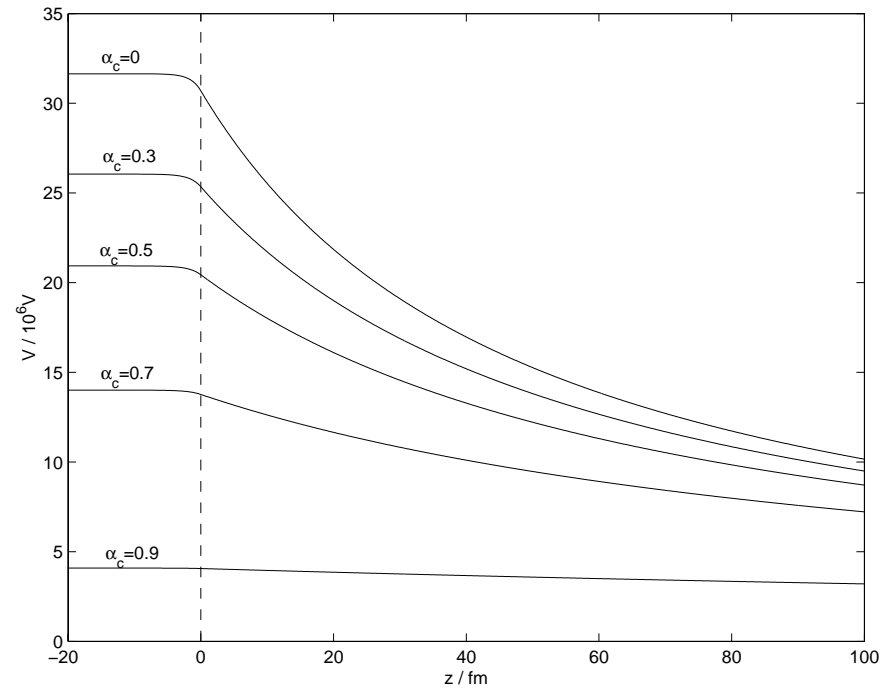

Fig. 1. Electric potential as a function of $z$ with $\alpha_{c}=0,0.3$, $0.5,0.7,0.9$.

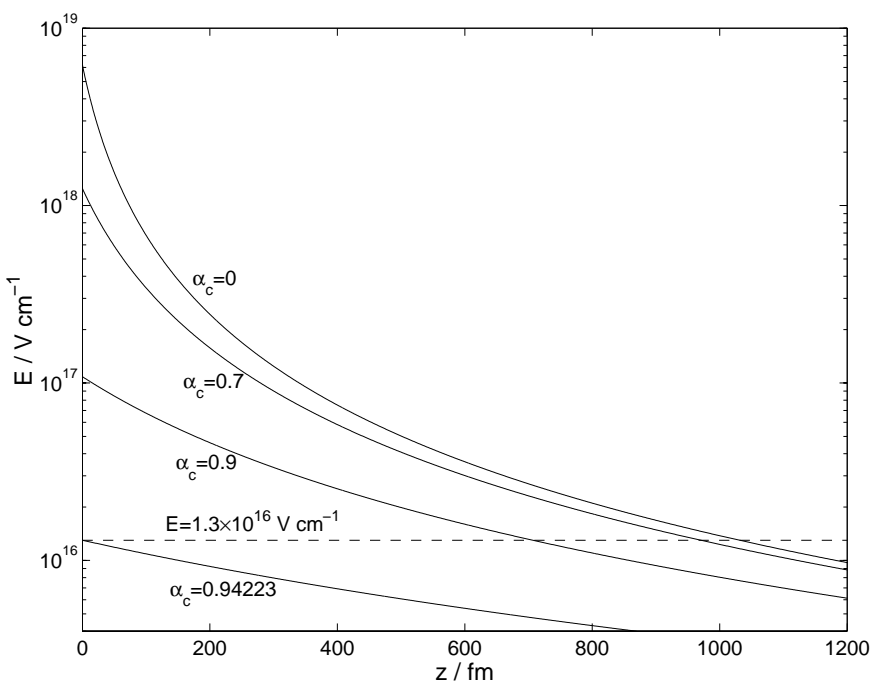

Fig. 2. Electric field changes slowly above the quark surface. The coupling constant $\alpha_{\mathrm{c}}$ is chosen to be $0,0.7,0.9,0.94$.

characteristics of a BSS for different values of $\alpha_{\mathrm{c}}$, assuming $^{2} \mu=300 \mathrm{MeV}, m_{\mathrm{s}}=200 \mathrm{MeV}$, and the renormalization point $\rho_{\mathrm{R}}=313 \mathrm{MeV}$. The quark and electron number densities $\left\{n_{\mathrm{i}}\right\}$ when $z \rightarrow-\infty$, as well as potentials of $V_{0}$ and $V_{\mathrm{c}}$, are listed in Table 1 for $\alpha_{\mathrm{c}}=$ $0,0.1,0.3,0.5,0.7,0.9$, respectively. The electric potential $V$ and field $E$ are also plotted in Figs. 1-3 for this case. We see from Fig. 1 that the potential curve becomes flatter when the coupling constant $\alpha_{\mathrm{c}}$ increases. Also it should be noted from Figs. 2 and 3 that the electric field $E$ changes rather slowly for $z>0$, but varies rapidly for $z<0$. This indicates the non-symmetric nature of the electric field with respect to the quark surface of $z=0$.

Secondly, we investigate numerically the thickness, $\Delta r_{\mathrm{E}}$, of the electron layer where pairs can be created,

\footnotetext{
${ }^{2}$ Generally there is a relation between $\mu$ and $B, \mu=\mu(B)$. In this assumption $B^{1 / 4} \sim 145 \mathrm{MeV}$.
}

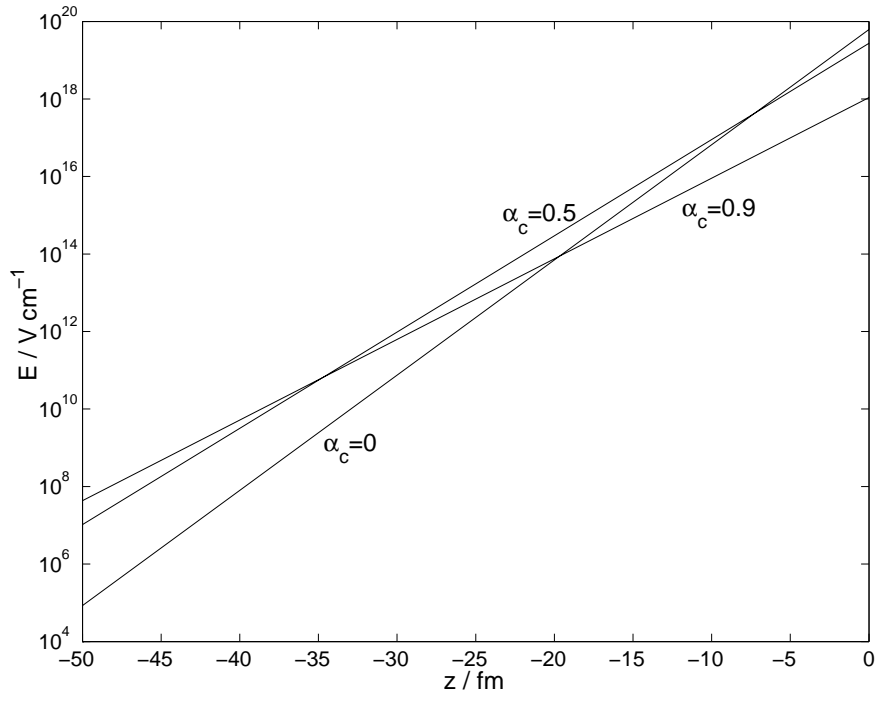

Fig. 3. Electric field varies sharply below the quark surface. $\alpha_{\mathrm{c}}=0,0.5,0.9$.

as functions of the bag parameters $m_{\mathrm{s}}, B$, and $\alpha_{\mathrm{c}}$. The results are shown in Fig. 4. It is found that the Usov mechanism can work for bag parameters within a rather wide range. The thickness $\Delta r_{\mathrm{E}}$ could be large enough (i.e., $\Delta r_{\mathrm{E}} \gtrsim 500 \mathrm{fm}$ ) as long as (1) $B$ is not too large and $m_{\mathrm{s}}$ is not too small, or (2) $B$ is not too small and $m_{\mathrm{s}}$ is not too large. Increasing $\alpha_{c}$ has an adverse influence on the Usov mechanism. For instance in Fig. 2, the field $E$ cannot exceed the critical field $E_{\mathrm{c}}=1.3 \times 10^{16} \mathrm{~V} / \mathrm{cm}$ (necessary for pair production) if $\alpha_{\mathrm{c}}>0.94$. Nevertheless previous dynamical calculations show that $\alpha_{\mathrm{c}} \gtrsim 0.9$ is less possible for stable strange quark matter. In conclusion we could expect that the pair emission process proposed by Usov might appear in nature for acceptable bag parameters.

\section{Conclusion and discussion}

We have improved the calculation of the electric characteristics of bare strange stars with the inclusion of boundary effects (i.e., the effects of non-local neutrality near and below the quark surface). From our calculation, we find that the Usov mechanism can work for bag parameters within a rather wide range.

As shown in Table 1 , both $V_{\mathrm{c}}$ and $V_{0}$, as well as their very small difference, decrease as $\alpha_{\mathrm{c}}$ increases. Our results on the electric potential $V(z)$ for $z<0$ is quite different to the previous calculation given by Alcock et al. (1986) where the boundary effects were not included (see Fig. 1). It is shown that the strong electric field resides only about $\sim 10$ fm below the quark surface (see Fig. 3), rather than $\sim 10^{2}$ fm obtained by Alcock et al. (1986).

We can proof that $E$ is almost an exponential function of $z$ below the quark surface. We denote the right hand side of Eq. (6) as $f(V)$. As $z \rightarrow-\infty$, namely $V \rightarrow V_{0}$, we have $f(V) \rightarrow 0$. Approximating $f(V)$ as $f^{\prime}\left(V_{0}\right)\left(V-V_{0}\right)$, we can obtain $E=-\sqrt{f^{\prime}\left(V_{0}\right)} \exp \left[\sqrt{f^{\prime}\left(V_{0}\right)}\right] z$ and $V=$ $V_{0}-\exp \left[\sqrt{f^{\prime}\left(V_{0}\right)} z\right]$ for $z<0$. 

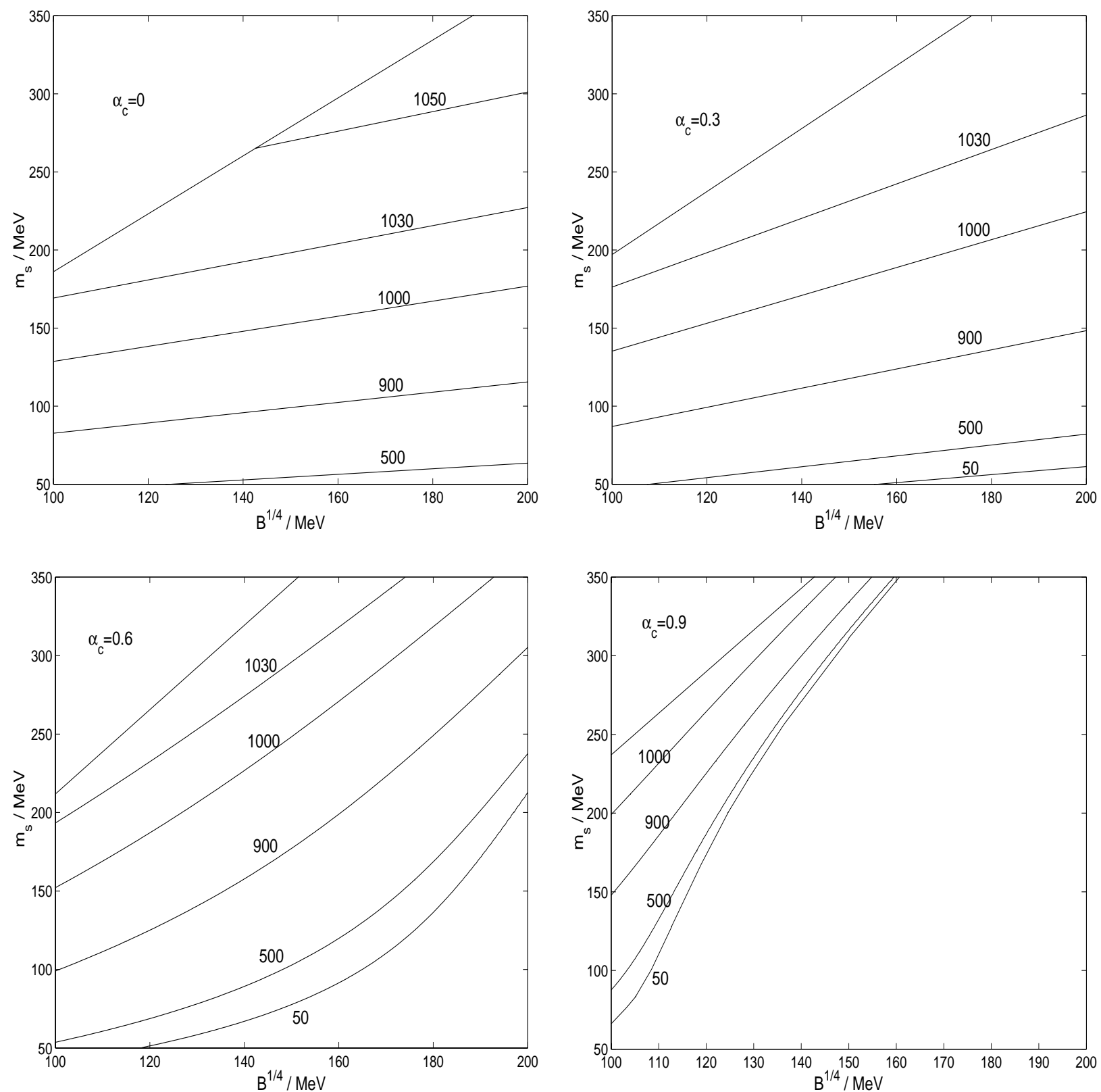

Fig. 4. A set of calculated thickness $\Delta r_{\mathrm{E}}$, as functions of bag parameters of $B$ and $m_{\mathrm{s}}$, for different coupling constants $\alpha_{\mathrm{c}}=0$, $0.3,0.6,0.9$. Indicated numbers denote the correspondence values of $\Delta r_{\mathrm{E}}$.

Acknowledgements. This work is supported by National Nature Sciences Foundation of China (10173002) and the Special Funds for Major State Basic Research Projects of China (G2000077602). The authors sincerely thank Dr. Shuangnan Zhang for his comments and the improvement of the language.

\section{References}

Alcock, C., Farhi, E., \& Olinto, A. 1986, ApJ, 310, 261 Day, M., Bombaci, I., Dey, J., Ray, S., \& Samanta, B. C. 1998, Phys. Lett. B 438, 123

Jensen, D. M., \& Madsen, J. 1996, Phys. Rev. D 53, R4712 Lugones, G., \& Benvenuto, O. G. 1995, Phy. Rev. D 52, 1276 Phukon, T. C. 2000, A\&A, 355, 1009

Huang, Y. F., \& Lu, T. 1997, A\&A, 325, 189
Kettner, Ch., Weber, F., Weigel, M. K., \& Glendenning, N. K. 1995, Phys. Rev. D 51, 1440

Martemyanov, B. V. 1992, Phys. Rev. D 49, 4293

Usov, V. V. 1998, Phys. Rev. Lett., 80, 230

Usov, V. V. 2001a, invited talk at the Conf. on Compact Stars in the QCD Phase Diagram, Copenhagen, Aug. 15-18 [astro-ph/0111442]

Usov, V. V. 2001b, ApJ, 550, L179

Usov, V. V. 2001c, Phys. Rev. Lett., 87, 021101

Usov, V. V. 2001d, ApJ, 559, L135

Xu, R. X., \& Qiao, G. J. 1999, Chin. Phys. Lett., 16, 778

Xu, R. X., \& Qiao, G. J. 1998, Chin. Phys. Lett., 15, 934

Xu, R. X., Qiao, G. J., \& Zhang, B. 1999, ApJ, 522, L109

Xu, R. X., Zhang, B., \& Qiao, G. J. 2001, Astroparticle Phys., 15,101

Zdunik, J. L., Haensel, P., \& Gourgoulhon, E. 2001, A\&A, 372, 535 